\title{
Physiotherapy practice in South African intensive care units
}

\author{
M Lottering, MSc (Physiotherapy); H van Aswegen, $\mathrm{PhD}$ \\ Department of Physiotherapy, School of Therapeutic Sciences, Faculty of Health Sciences, University of the Witwatersrand, Johannesburg, South Africa
}

Corresponding author: $H$ van Aswegen (helena.vanaswegen@wits.ac.za)

\begin{abstract}
Background. Physiotherapists are integral members of the interprofessional team that provides care and rehabilitation for patients in intensive care units (ICUs).

Objectives. To describe the current practice of physiotherapists in ICUs, determine if physiotherapists' practice has changed since a previous report and determine if practice is evidence based.

Methodology. A questionnaire was content validated and made available electronically and in hard copy. Physiotherapists who work in ICUs in public or private sector hospitals or who are members of the South African Society of Physiotherapy were identified and invited to participate. Results. Survey response rate was 33.9\%. Patient assessment techniques performed 'very often' included ICU chart assessment ( $n=90,83.3 \%$ ), chest auscultation $(n=94,81.8 \%)$ and cough effort $(n=81,75 \%)$. Treatment techniques performed 'very often' included manual chest clearance $(n=101,93.5 \%)$, in-bed mobilisation and positioning $(n=91,84.3 \% ; n=91,84.3 \%$, respectively), airway suctioning $(n=89,82.4 \%)$, out-of-bed mobilisation $(n=84,77.8 \%)$, deep breathing exercises $(n=83,76.9 \%)$ and peripheral muscle-strengthening exercises $(n=72,73.1 \%)$. More respondents used intermittent positive pressure breathing $(57$ v. $28 \%, p=0.00)$, used adjustment of mechanical ventilation (MV) settings $(30 \mathrm{v} .15 \%, p=0.01)$, were involved with weaning patients from MV ( $42 \mathrm{v}$. $19 \%, p=0.00)$ and used incentive spirometry $(76 \mathrm{v} .46 \%, p=0.00)$ than reported previously. More respondents performed suctioning ( 99 v. $70 \%, p=0.00)$, extubation ( 60 v. $25 \%, p=0.00)$ and adjustment of MV settings ( $30 \mathrm{v} .12 \%, p=0.02$ ) than reported internationally.

Conclusion. Physiotherapy practice in ICUs is evidence based. Care focuses largely on mobilisation, exercise therapy and multimodality respiratory therapy.
\end{abstract}

S Afr J Crit Care 2016;32(1):11-16. DOI:10.7196/SAJCC.2016.v3211.248

Research activity in critical care literature is currently focused on early rehabilitation of patients with critical illness and its effects on length of stay, number of ventilator-free days and functional outcomes. ${ }^{[1,2]}$ Physiotherapists are integral members of the interprofessional team that provides care and rehabilitation to patients with critical illness, on an international and national level. ${ }^{[1,3,4]}$ Recently, there has been a drive to establish minimum standards of clinical practice for physiotherapists in South African (SA) intensive care units (ICUs) to ensure that safe and effective patient care is provided. ${ }^{[4]}$ Few reports on physiotherapy practice in ICU could be sourced. In 2000, Norrenberg and Vincent ${ }^{[5]}$ conducted a study to establish the profile of physiotherapists working in ICUs in Europe. Van Aswegen and Potterton ${ }^{[6]}$ subsequently amended the survey questionnaire compiled by the aforementioned authors for use in an SA setting and conducted a pilot survey to determine the scope of practice of physiotherapists in ICUs. Content validation of the modified questionnaire was not carried out at the time and, therefore, the reported results were preliminary. Defining the current practice of physiotherapists working in ICUs is important to assist with the development of clinical practice guidelines and minimum standards of physiotherapy practice. It was therefore decided to conduct a nationwide survey to: (i) determine the current practice of physiotherapists in SA ICUs; (ii) determine if physiotherapists' practice in ICUs had changed since the previous report; and (iii) validate the survey questionnaire. In addition, SA physiotherapists' practice in ICU was compared with that reported in critical care and rehabilitation literature, to determine if current practice is evidence based.

\section{Method}

A cross-sectional, quantitative, descriptive, survey-based study was performed. Ethical clearance was sought and obtained from the University of the Witwatersrand Human Research Ethics (Medical) Committee (clearance no.: M130131) prior to commencement. The first phase of the study consisted of content validation of the questionnaire used by van Aswegen and Potterton. ${ }^{[6]}$ The questionnaire and survey objectives were sent electronically to a group of five physiotherapists experienced in working in ICUs. They were asked to review the documents prior to a meeting scheduled with the authors. At the meeting, questions that were deemed unsuitable were either adjusted or removed from the questionnaire and additional questions were added where indicated. The amended questionnaire was emailed back to the group members for further scrutiny. A few minor adjustments were then suggested and made by the authors. After consensus was reached on the content of the final version of the questionnaire, it was loaded onto SurveyMonkey for the second phase of the study. The final version of the questionnaire consisted of the following sections: participant demographics, ICU type in which physiotherapists work, patient referral method, after-hours service provision, assessment and treatment techniques used in patient management, participation in interprofessional team meetings and professional development activities.

Physiotherapists with work experience of 3 years or more in adult ICUs in public or private sector hospitals or who were members of the Cardiopulmonary Physiotherapy Rehabilitation Group (CPRG) of the SA Society of Physiotherapy (SASP) were contacted in 2013 and invited to participate in the survey. Potential participants were identified using the following methods:

(i) The Department of Health was contacted to obtain a list of all public sector hospitals in SA. Using this list, all physiotherapy heads of departments (HODs) in public sector hospitals that had ICUs were contacted and informed of the aims of and inclusion criteria for the survey.

(ii) A list of hospitals belonging to the Life, Mediclinic and Netcare groups was obtained from their respective websites and hospitals with ICUs were contacted. The unit manager of the ICU was contacted for 
details of the physiotherapy private practices working in their unit. After agreement was received from practice owners for their details to be shared, unit managers passed the information to the authors who contacted them regarding the aims of and inclusion criteria for the survey.

(iii) The chairperson of the CPRG of the SASP was contacted and informed of the aims of and inclusion criteria for the survey. The chairperson was asked to invite members of the CPRG to participate in the survey.

Practice owners and HODs were asked to discuss the survey with their staff. The contact details of those who fitted the inclusion criteria and agreed to participate were passed on to the authors. An electronic link to the survey was sent to these physiotherapists. Alternatively, hard copies of the survey information sheet, inclusion criteria, questionnaire and self-addressed return envelopes were distributed to those who did not have access to the electronic link. All hard copy questionnaires were coded. Participants were given 3 months, from May 2013, to complete the questionnaire, and consent was implied. One and 2 months after initiation of the survey, a blanket reminder email was sent out to all participants who were initially emailed with the electronic link, to remind them to complete the survey and to thank those who had already done so. The HODs of hospitals that were sent postal questionnaires were phoned at the same intervals and asked to remind their staff to complete and return the questionnaires; those who had already completed questionnaires were thanked.

\section{Statistical analysis}

Categorical data were summarised as frequencies and percentages in text and illustrative tables. Comparisons of findings from the current survey with those reported by van Aswegen and Potterton, ${ }^{[6]}$ and Norrenberg and Vincent ${ }^{[5]}$ were made using the $\chi^{2}$ test or in narrative form where statistical comparisons were not possible. The denominator for the $\chi^{2}$ test was Yes/No, related to each treatment technique used as reported in the current survey and results reported by the other two surveys. ${ }^{[5,6]} \mathrm{A} p$-value of $\leq 0.05$ was considered statistically significant. SPSS version 22.0 (IBM Corp., USA) was used for statistical analyses.

\section{Results}

The authors identified 71 public hospitals that had ICUs at the time of the survey. There were 64 Life, 49 Mediclinic and 56 Netcare hospitals

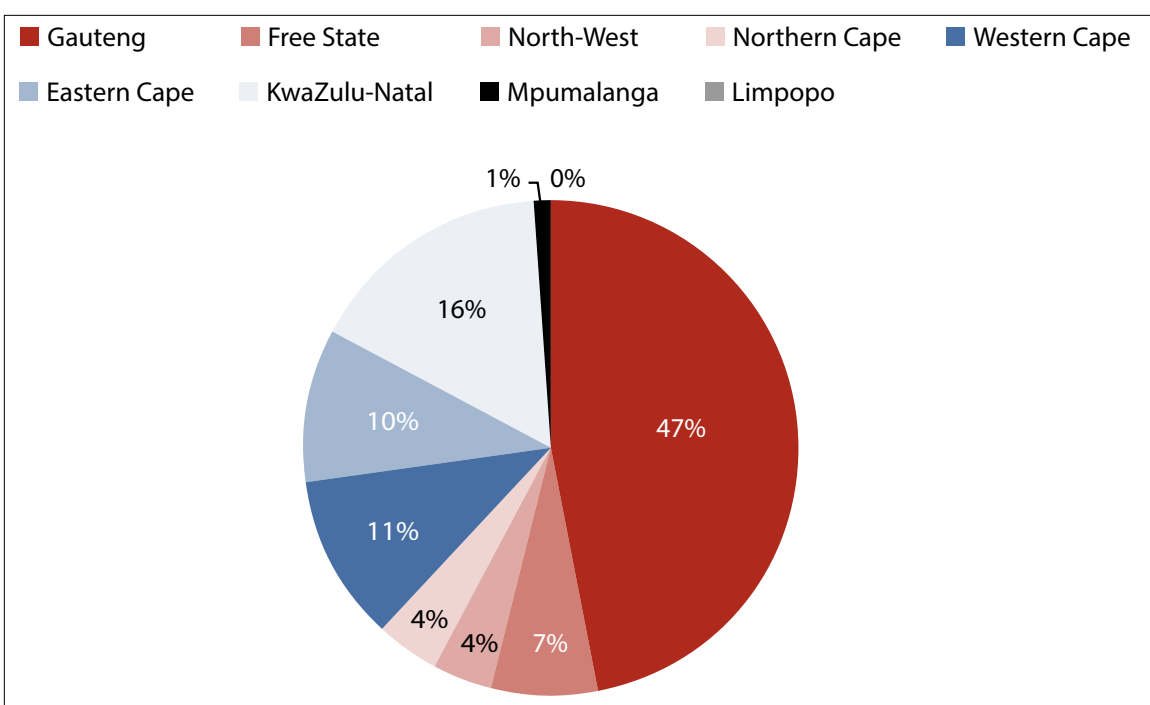

Fig. 1. Geographical representation of respondents $(n=108)$.

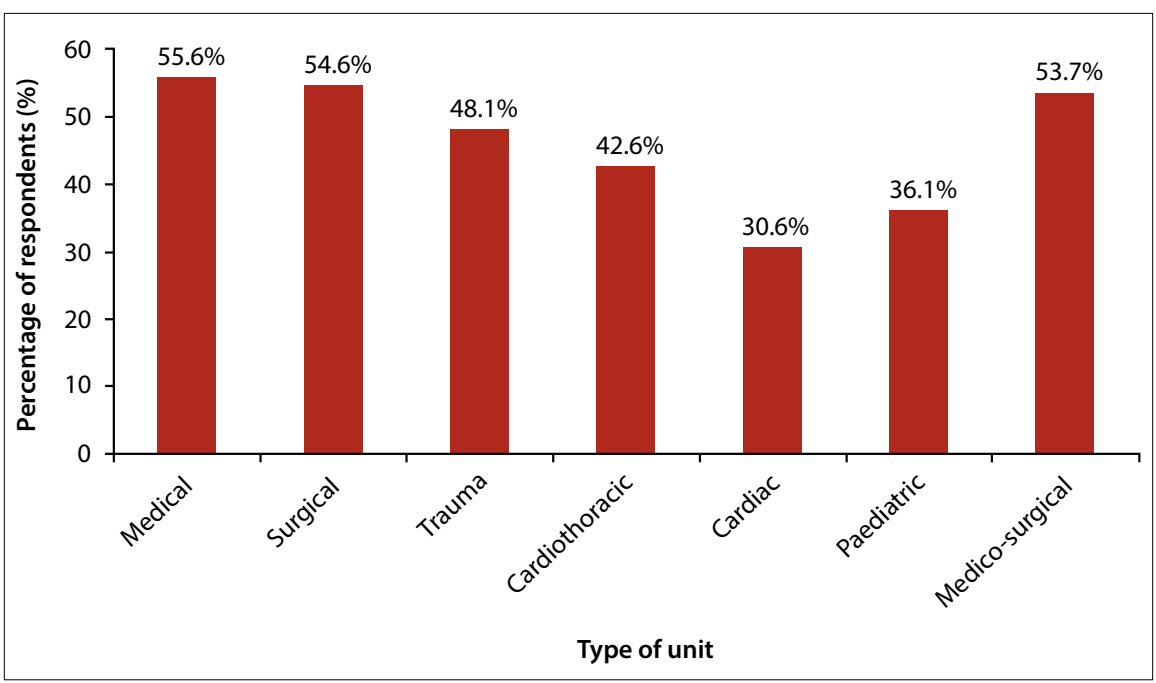

Fig. 2. Type of ICU in which respondents worked.

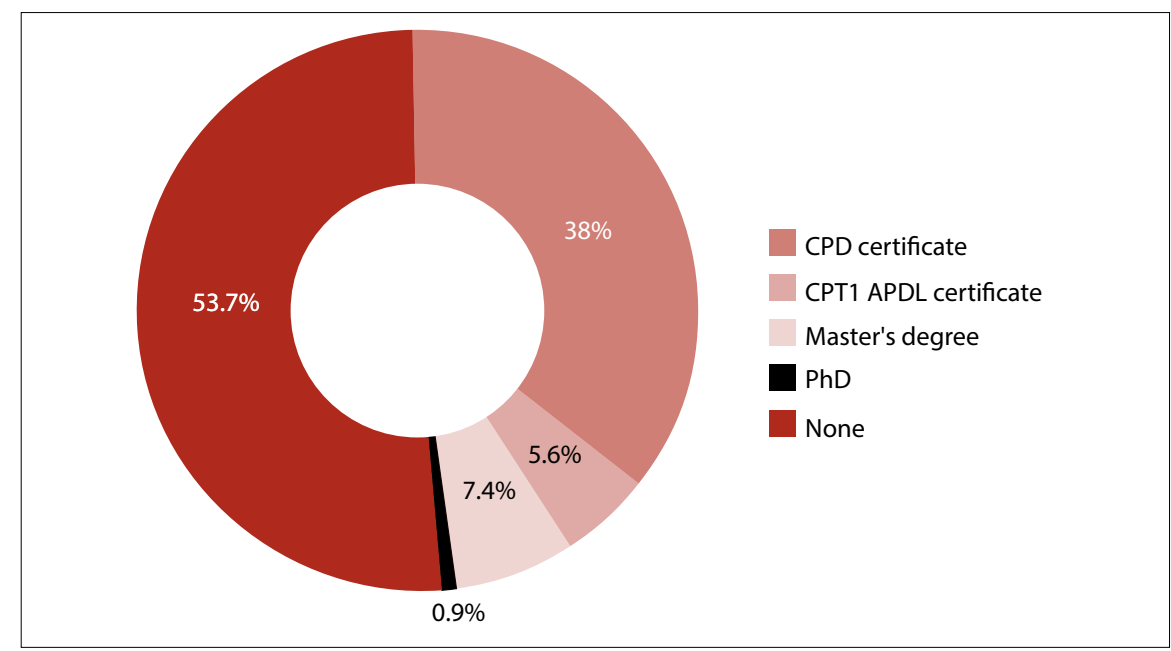

Fig. 3. Percentage of respondents who held any type of postgraduate cardiopulmonary physiotherapy qualification.

in SA at the time of the survey, of which 49 Life, 32 Mediclinic and 44 Netcare hospitals had ICUs with physiotherapy service provision. These 125 private hospitals were contacted by the authors, and contact details of 154 private physiotherapy practices were obtained. A total of 319 questionnaires were sent out ( $n=252$ electronic and $n=67$ postal). Eighty- 
five electronic responses were received and 23 postal questionnaires were returned (response rates of $33.7 \%$ and $34.3 \%$, respectively) with a combined response rate of $33.9 \%(n=108)$.

Physiotherapists working in private sector hospitals made up $60.2 \%$ $(n=65)$ of respondents. Responses were received from physiotherapists in all the SA provinces with the exception of Limpopo Province (Fig. 1). A large number of respondents $(n=51,47.2 \%)$ were from
Gauteng. The majority of respondents worked in medical, surgical or combined medico-surgical ICUs (Fig. 2). Fifty-six respondents (46\%) held a postgraduate qualification related to cardiopulmonary physiotherapy (Fig. 3). In most cases, respondents indicated that patients in ICU were referred for physiotherapy by doctors or nurses $(n=59,54 \%)$; however, some reported that the physiotherapist working in the unit screened the patients to determine if they

Table 1. Frequency with which respondents used assessment techniques in ICU, ${ }^{\star} n$

\begin{tabular}{|c|c|c|c|c|c|}
\hline Assessment technique & Never & Almost never & Sometimes & Fairly often & Very often \\
\hline Auscultation & 0 & 1 & 2 & 11 & 94 \\
\hline ICU chart review & 1 & 0 & 5 & 12 & 90 \\
\hline Strength of cough effort & 4 & 3 & 7 & 13 & 81 \\
\hline $\mathrm{X}$-ray/Computed tomography scan & 0 & 0 & 7 & 28 & 73 \\
\hline Arterial blood gas analysis & 3 & 6 & 15 & 24 & 60 \\
\hline Readiness for mobilisation using specific criteria & 7 & 10 & 15 & 33 & 43 \\
\hline Need for humidification & 22 & 13 & 25 & 20 & 28 \\
\hline Peripheral muscle strength (dynamometry or Medical Research Council scale) & 30 & 16 & 21 & 19 & 22 \\
\hline Respiratory muscle strength (MIP) & 25 & 24 & 25 & 18 & 16 \\
\hline Percussion note to assess quality of lung tissue & 29 & 24 & 24 & 17 & 14 \\
\hline Calculation of lung compliance & 39 & 36 & 18 & 8 & 7 \\
\hline Calculation for the presence of hypoxaemia & 45 & 29 & 20 & 9 & 5 \\
\hline
\end{tabular}

Table 2. Frequency with which respondents used various treatment techniques in their management of critically ill patients, $n$

\begin{tabular}{|c|c|c|c|c|c|}
\hline Physiotherapy technique & Never & Almost never & Sometimes & Fairly often & Very often \\
\hline Manual chest clearance techniques (percussions, vibrations, shaking) & 2 & 0 & 1 & 4 & 101 \\
\hline Mobilising a patient in bed & 0 & 1 & 0 & 16 & 91 \\
\hline Positioning a patient in bed & 0 & 0 & 5 & 12 & 91 \\
\hline Airway suctioning & 1 & 0 & 2 & 16 & 89 \\
\hline Mobilising a patient out of bed & 0 & 2 & 6 & 16 & 84 \\
\hline Positioning a patient out of bed & 0 & 3 & 8 & 18 & 79 \\
\hline Peripheral muscle-strengthening exercises & 2 & 0 & 5 & 29 & 72 \\
\hline Postural drainage/modified postural drainage & 2 & 2 & 12 & 25 & 67 \\
\hline Active cycle of breathing techniques & 5 & 6 & 15 & 26 & 56 \\
\hline Nebulisation & 2 & 4 & 7 & 43 & 52 \\
\hline Blow bottle & 20 & 11 & 16 & 30 & 31 \\
\hline Blowing up a glove & 33 & 6 & 31 & 18 & 20 \\
\hline Inspiratory muscle training (threshold device/devices by other manufacturers) & 31 & 23 & 23 & 15 & 16 \\
\hline Manual hyperinflation (ambubagging) & 14 & 26 & 33 & 20 & 15 \\
\hline IPPB & 27 & 19 & 28 & 21 & 13 \\
\hline Active involvement in weaning a patient from $\mathrm{MV}$ & 39 & 24 & 16 & 16 & 13 \\
\hline Adjustment of MV settings for respiratory muscle training & 53 & 23 & 15 & 13 & 4 \\
\hline Implementation and supervision of non-invasive ventilator support (CPAP, BiPAP) & 55 & 22 & 13 & 15 & 3 \\
\hline Flutter device & 46 & 31 & 17 & 11 & 3 \\
\hline
\end{tabular}


were suitable for physiotherapy intervention $(n=49,45 \%)$. An after-hours physiotherapy service was provided by $72 \%(n=78)$ of respondents to ICUs during weekdays. The majority of respondents $(n=105,97 \%)$ provided weekend physiotherapy services to their ICUs.

Respondents were asked how often they used various methods of assessment to determine patient suitability for physiotherapy intervention and how often they used various treatment techniques in their patient management. They could select responses indicating either 'never', 'almost never', 'sometimes', 'fairly often' or 'very often'. Responses related to assessment are summarised in Table 1 and those related to treatment in Table 2. The most frequently used assessment techniques included ICU chart assessment $(n=90,83 \%)$, chest auscultation $(n=94,82 \%)$ and assessment of strength of cough effort $(n=81,75 \%)$. Assessment techniques that were 'almost never' or 'never' used included calculation of lung compliance $(n=75,69 \%)$, calculation of hypoxaemia $(n=74,69 \%)$ and assessment of patient readiness for weaning $(n=63$, $58 \%)$. Objective outcome measures such as MIP, RSBI, dynamometry or Medical Research Council scale were more often not used. Treatment techniques performed by respondents 'very often' included manual chest clearance techniques $(n=101,94 \%)$, mobilising a patient in bed $(n=91,84 \%)$, positioning a patient in bed $(n=91,84 \%)$, airway suctioning $(n=89,82 \%)$, mobilising a patient out of bed $(n=84,78 \%)$, deep breathing exercises $(n=83,77 \%)$ and peripheral muscle-strengthening exercises $(n=79,73 \%)$. Treatment techniques that respondents 'never' or 'almost never' used included the flutter device $(n=77,71 \%)$, implementation and supervision of noninvasive ventilation support ( $n=77,71 \%)$ and adjustment of mechanical ventilation (MV) settings for respiratory muscle training ( $n=76,70 \%)$.

Fifty-six percent $(n=60)$ of respondents attended ward rounds in the ICU on a daily or weekly basis. Forty-five percent $(n=49)$ of respondents had attended an ICUrelated continuous professional development activity within the last year. Thirty-four percent $(n=37)$ of respondents were involved in student training and 33\% $(n=36)$ were involved in training of other members of the interprofessional team in the ICU. A large number of respondents were involved with the inservice training of colleagues, such

Table 3. A comparison of physiotherapy techniques used by the respondents in the study by Norrenberg and Vincent ${ }^{[5]}$ and the current study

\begin{tabular}{|c|c|c|c|c|c|}
\hline \multirow[b]{2}{*}{ Treatment technique } & \multicolumn{2}{|c|}{$\begin{array}{l}\text { Norrenberg } \\
\text { and Vincent } \\
\text { (Europe, 2000) }\end{array}$} & \multicolumn{2}{|c|}{$\begin{array}{l}\text { Current study } \\
(\mathrm{SA}, 2013)\end{array}$} & \multirow[b]{2}{*}{$p$-value } \\
\hline & Yes, \% & No, \% & Yes, \% & No, $\%$ & \\
\hline Respiratory treatment & 98 & 2 & 98 & 2 & 1 \\
\hline Suctioning & 70 & 16 & 99 & 1 & 0.00 \\
\hline IPPB/NIPPV & 46 & 29 & 57 & 43 & 0.56 \\
\hline Intubation & 1 & 90 & 1 & 99 & 0.95 \\
\hline Extubation & 25 & 50 & 60 & 40 & 0.00 \\
\hline Adjustment of MV & 12 & 65 & 30 & 70 & 0.02 \\
\hline Weaning from MV & 22 & 56 & 42 & 58 & 0.06 \\
\hline Mobilising & 100 & 0 & 99 & 1 & 0.32 \\
\hline Positioning & 90 & 1 & 99 & 1 & 0.95 \\
\hline
\end{tabular}

Table 4. A comparison of physiotherapy techniques used by the respondents in the study by van Aswegen and Potterton ${ }^{[6]}$ and the current study

\begin{tabular}{|c|c|c|c|c|c|}
\hline \multirow[b]{2}{*}{ Treatment technique } & \multicolumn{2}{|c|}{$\begin{array}{l}\text { Van Aswegen } \\
\text { and Potterton } \\
(\mathrm{SA}, 2005)\end{array}$} & \multicolumn{2}{|c|}{$\begin{array}{l}\text { Current study } \\
(\mathrm{SA}, 2013)\end{array}$} & \multirow[b]{2}{*}{$p$-value } \\
\hline & Yes, \% & No, $\%$ & Yes, \% & No, $\%$ & \\
\hline Respiratory treatment & 98 & 2 & 98 & 2 & 1 \\
\hline Suctioning & 98 & 2 & 99 & 1 & 0.56 \\
\hline IPPB & 28 & 72 & 57 & 43 & 0.00 \\
\hline Intubation & 2 & 98 & 1 & 99 & 0.56 \\
\hline Extubation & 65 & 35 & 60 & 40 & 0.46 \\
\hline Adjustment of MV & 15 & 85 & 30 & 70 & 0.01 \\
\hline Weaning from MV & 19 & 81 & 42 & 58 & 0.00 \\
\hline Mobilising & 98 & 2 & 99 & 1 & 0.56 \\
\hline Positioning & 95 & 5 & 99 & 1 & 0.10 \\
\hline Blow bottle & 70 & 30 & 71 & 29 & 0.88 \\
\hline MHI & 75 & 25 & 63 & 37 & 0.07 \\
\hline IS & 46 & 54 & 76 & 24 & 0.00 \\
\hline
\end{tabular}

as training junior physiotherapists to work safely in the ICU $(n=51,47 \%)$ and training other physiotherapists at their hospital or private practice to work safely in the ICU $(n=55,50 \%)$.

Comparisons were made between physiotherapy treatment techniques used in this survey and those reported by Norrenberg and Vincent ${ }^{[5]}$ (Table 3). More SA respondents performed suctioning $(99 \mathrm{v}$. $70 \%, p=0.00$ ), extubation ( 60 v. $25 \%, p=0.00$ ) and adjustment of MV settings (30 v. $12 \%$, $p=0.02)$ than their European counterparts. Similarly, comparisons were made between physiotherapy treatment techniques used in this survey and those reported by van Aswegen and Potterton ${ }^{[6]}$ (Table 4). More respondents in the current survey used IPPB ( 57 v. $28 \%, p=0.00$ ), performed adjustment of MV settings ( 30 v. $15 \%, p=0.01$ ), were involved with weaning patients from MV (42 v. $19 \%, p=0.00)$ and used IS (76 v. $46 \%, p=0.00)$ in patient management than reported in the 2005 survey. ${ }^{[5]}$

\section{Discussion}

Immobility, bed rest and inflammation during critical illness result in impaired ventilation, decreased lung compliance and increased airway resistance. These conditions lead to respiratory system dysfunction, muscle protein breakdown and weakness, and subsequent neuromusculoskeletal system dysfunction. ${ }^{[3]}$ As a result, physiotherapists 
are involved in the prevention and treatment of respiratory and neuromusculoskeletal conditions of patients with critical illness. ${ }^{[1,3]}$ This report provides updated information on physiotherapy practice in SA adult ICU settings and is timely, in light of a recent drive to develop minimum standards for physiotherapy practice in ICU. ${ }^{[4]}$

Clinical decision-making regarding rehabilitation interventions in the ICU is based on multisystem assessment of the critically ill patient to identify potential problems and precautions, and contraindications to such interventions. ${ }^{[7,8]}$ Most of the respondents in this survey frequently conducted ICU chart assessment, auscultation, assessment of cough effort, thoracic expansion, assessment of radiological investigations and patient readiness for mobilisation. This is in keeping with recommendations made by others on patient assessment in $\operatorname{ICU}^{[4,7,8]}$ and supports the importance of individualised patient management in ICU. Physiotherapy patient assessment was not reported on in the surveys by Norrenberg and Vincent ${ }^{[5]}$ or van Aswegen and Potterton ${ }^{[6]}$ and therefore comparison with current results cannot be made.

Most respondents reported that they frequently performed mobilisation of patients in and out of bed and peripheral musclestrengthening exercises. This is in keeping with the statement of Hanekom et al. ${ }^{[4]}$ that physiotherapists are rehabilitation experts who form an integral part of the interprofessional team in ICU. The indisputable role for physiotherapy in the ICU setting is confirmed by strong evidence that demonstrates the beneficial effects of early mobilisation and strengthening exercises on ICU and hospital length of stay, number of ventilator-free days and functional outcomes. ${ }^{[1,2]}$

The use of outcome measures is important to determine patients' responses to treatment received and evaluate the usefulness of treatment techniques employed. ${ }^{[9,10]}$ Even though regular assessment of patient readiness for mobilisation was reported, it was interesting to find that respondents did not often use outcome measures such as dynamometry or the Medical Research Council scale to assess patients' peripheral muscle strength. Calculation for the presence of hypoxaemia or decreased lung compliance was rarely done. Poor oxygenation and restricted lung ventilation is known to affect a patient's respiratory reserve and their ability to perform exercise and mobilisation. Time constraints and lack of equipment were highlighted as factors that influence physiotherapists' use of outcome measures in daily practice. ${ }^{[9]}$ The authors speculate that these may have been contributory factors to the results of the current survey but this would require further investigation.

Assessment of patient readiness to wean from MV and maximal inspiratory pressure to assess respiratory muscle strength was done less often. Respondents reported using active cycle of breathing techniques or deep breathing exercises frequently during patient management in the ICU, but only a few used inspiratory muscle trainer devices or adjustment (temporary) of mechanical ventilator settings for respiratory muscle training. They reported limited active involvement in weaning of patients from MV. This is not in keeping with the report by Plani et al., ${ }^{[11]}$ which showed that physiotherapists' involvement in a weaning and extubation protocol implemented in the management of critically ill trauma patients in an SA ICU setting resulted in a clinically significant reduction in MV time. The use of respiratory muscle trainer devices has been shown to significantly increase maximal inspiratory pressure ${ }^{[12,13]}$ and tidal volume ${ }^{[13]}$ and shorten time to weaning from $\mathrm{MV}^{\left[{ }^{[12]}\right.}$ The majority of respondents reported being involved with extubation of patients and more respondents in the current survey reported involvement with adjustment of mechanical ventilator settings and weaning of patients from MV than reported previously ${ }^{[6]}$ However, reasons for continued limited active involvement of physiotherapists in weaning of patients from MV require further exploration.

Pulmonary hygiene in the form of nebulisation, manual chest clearance techniques, postural drainage/modified postural drainage positions, positioning a patient in bed and suctioning were performed frequently, whereas manual hyperinflation was performed less often. These findings are in keeping with the use of multimodality respiratory physiotherapy in the care of critically ill patients described by Stiller. ${ }^{[1]}$ Multimodality respiratory physiotherapy, a combination of techniques including positioning, chest-wall vibrations, percussions, manual hyperinflation and suction, ${ }^{[1,14,15]}$ has been shown to reduce the incidence of ventilator-associated pneumonia ${ }^{[14]}$ and shorten duration of MV and ICU length of stay in previous reports. ${ }^{[15]}$ The selective use of manual hyperinflation in patient care is in keeping with research evidence, as this technique is associated with side-effects such as marked haemodynamic changes in the form of decreased cardiac output in some critically ill patients, and increased intracranial pressure in traumatic brain injury patients. ${ }^{[3]}$ Alternatively, physiotherapists' level of confidence in the application of manual hyperinflation as part of patient management might have influenced the results and would need further exploration. Assessment of patients' need for humidification was done less often by respondents in this survey. Patients who receive supplemental oxygen via non-invasive ventilation or through invasive ventilation via an artificial airway require adequate humidification to prevent secretions from becoming too viscous, forming mucus plugs in distal airways and resulting in lung volume loss or infection; ${ }^{[16,18]}$ therefore, humidification therapy and regular assessment for changes in patient humidification needs should form part of daily practice in the ICU. Oscillatory positive expiratory pressure (PEP) in the form of a blow bottle seems to be a popular treatment intervention for physiotherapists in SA, as a large number of respondents in the current and $2005^{[6]}$ surveys reported using it as a treatment strategy. Limited evidence exists to support the use of PEP over other breathing exercises in patients following abdominal or thoracic surgery. ${ }^{[19]}$ More respondents in the current survey used IPPB and IS as part of patient treatment in ICU than those in the 2005 survey ${ }^{[6]}$ IS is frequently used by others in the postoperative setting for lung volume recruitment in spontaneously breathing patients. ${ }^{[3]} \mathrm{A}$ limited number of respondents used IPPB or blowing up a glove on a regular basis, which may reflect that limited research evidence exists to support their use in clinical practice, especially glove-blowing. The authors acknowledge, however, that varying availability of IPPB equipment in the clinical setting may have influenced results.

Compulsory training in theoretical and clinical aspects of care of the critically ill patient is offered to all undergraduate physiotherapy students at the eight universities in SA. Postgraduate physiotherapy training in the field of critical care is voluntary, ${ }^{[4]}$ which offers an explanation for the relatively low number of respondents with any type of postgraduate qualification. In the survey by Van Aswegen and Potterton, ${ }^{[6]} 36 \%$ of respondents held a postgraduate qualification compared with $46 \%$ in the current survey, which indicates a growing interest among SA physiotherapists to build on the knowledge base obtained at undergraduate level to work safely and effectively with critically ill patients. A number of respondents indicated that they shared their knowledge through training of students, junior physiotherapists and other members of the ICU interprofessional team. The majority of respondents provided after-hours physiotherapy service to the ICU on 
weekdays. This was, however, less than the $82-94 \%$ of respondents who provided a weekday after-hours physiotherapy service to ICUs in the 2005 survey. ${ }^{[6]}$ Reasons for this reduced service provision are unclear. Physiotherapy service provision to the ICU over the weekend remains a priority of care, as almost all respondents provided such service in the current survey, similar to previous results $(96 \%) .{ }^{[6]}$ One area of concern is that only half of the respondents were involved in training of physiotherapists in their hospital to ensure that they work safely in ICU after hours; this requires further exploration.

\section{Study limitations}

The response rate of survey-based studies may influence the standard and quality of research; therefore, it is important to know if a surveybased study has a high non-response bias. A response rate of $60 \%$ should be the goal for all survey-based studies. ${ }^{[20]}$ The current study carries a $66 \%$ non-response bias and thus the results should be interpreted with caution.

Recently, emphasis has been placed on the use of validated outcome measurement tools such as the Chelsea Critical Care Physical Assessment (CPAx) tool ${ }^{[21]}$ and Physical Function in ICU test (PFIT) ${ }^{[8]}$ to assess patient function in ICUs. The use of electrical muscle stimulation to prevent disuse muscle atrophy in critically ill patients is gaining popularity among clinicians and researchers. ${ }^{[1,3]}$ Future surveys on physiotherapy practice in SA ICUs should include these.

\section{Conclusion}

The care provided by physiotherapists to adult patients in SA ICUs consists mostly of exercise therapy, mobilisation and multimodality respiratory therapy. This is in keeping with international reports in the fields of critical care and rehabilitation. Future exploration of physiotherapists' role in weaning of patients from MV, use of functional outcome measures and electrical muscle stimulation in the critical care setting, as well as the training of staff for after-hours service provision, is recommended.
References

1. Stiller K. Physiotherapy in intensive care: An updated systematic review. Chest 2013;144(3):825847. DOI:10.1378/chest.12-2930

2. Cameron S, Ball I, Cepinskas G, et al. Early mobilisation in the critical care unit: A review of adult and pediatric literature. J Crit Care 2015;30:664-672. DOI:10.1016/j.jcrc.2015.03.032

3. Gosselink R, Clerckx B, Robeets C, Vanhullebusch T, Vanpee G, Segers J. Physiotherapy in the intensive care unit. Neth J Crit Care 2011;15(2):66-75.

4. Hanekom S, van Aswegen H, Plani N, Patman S. Developing minimum clinical standards for physiotherapy in South African intensive care units: The nominal group technique in action J Eval Clin Pract 2015;21(1):118-127. DOI:10.1111/jep.12257

5. Norrenberg M, Vincent JL. A profile of European intensive care unit physiotherapists. Intensive Care Med 2000;26(7):850-856. DOI:10.1007/s001340051292

6. Van Aswegen H, Potterton J. A pilot survey of the current scope of practice of South African physiotherapists in intensive care units. S Afr J Physiother 2005;61(1):17-21.

7. Gosselink R, Bott J, Johnson M, et al. Physiotherapy for adult patients with critical illness: Recommendations of the European respiratory society and European society of intensive care medicine task force on physiotherapy for critically ill patients. Intensive Care Med 2008;34(7):1188-1199.

8. Berney S, Haines K, Denehy L. Physiotherapy in critical care in Australia. Cardiopulm Phys Ther J 2012;23(1):19-25.

9. Maher C, Williams M. Factors influencing the use of outcome measures in physiotherapy management of lung transplant patients in Australia and New Zealand. Physiother Theory Pract 2005;21(4):201-217. DOI:10.1080/09593980500321093

10. Marques A, Bruto A, Barney A. Clinically useful outcome measures for physiotherapy airway clearance techniques: A review. Phys Ther Rev 2006;11:299-307. DOI:10.1179/108331906X163441

11. Plani N, Becker P, van Aswegen $\mathrm{H}$. The use of a weaning and extubation protocol to facilitate effective weaning and extubation from mechanical ventilation in patients suffering from traumatic injuries: A non-randomised experimental trial comparing a prospective to retrospective cohort. Physiother Theory Pract 2013;29(3):211-221.

12. Cader SA, Vale RG, Castro JC, et al. Inspiratory muscle training improves maximal inspiratory pressure and may assist weaning in older intubated patients: A randomised trial. J Physiother 2010;56(3):171-177.

13. Condessa RL, Brauner JS, Saul AL, et al. Inspiratory muscle training did not accelerate weaning from mechanical ventilation but did improve tidal volume and maximal respiratory pressures: A randomised trial. J Physiother 2010;59(2):101-107.

14. Ntoumenopoulos G, Presneill JJ, McElholum M, Cade JF. Chest physiotherapy for the prevention of ventilator-associated pneumonia. Intensive Care Med 2002;28(7):850-856. DOI:10.1007/ s00134-002-1342-2

15. Malkoç M, Karadibak D, Yildirim Y. The effect of physiotherapy on ventilator dependency and the length of stay in an intensive care unit. Int J Rehabil Res 2009;32(1):85-88.

16. Branson RD. Secretion management in the mechanically ventilated patient. Respir Care 2007;52(10):1328-1347

17. Solomita M, Palmer LB, Daroowalla F, et al. Humidification and secretion volume in mechanically ventilated patients. Respir Care 2009;54(10):1329-1335.

18. Esquinas Rodriguez AME, Scala R, Soroksky A, et al. Clinical review: Humidifiers during non-invasive ventilation - key topics and practical implications. Crit Care 2013;16(1):203-210. DOI:10.1186/cc10534

19. Orman J, Westerdahl E. Chest physiotherapy with positive expiratory pressure breathing after abdominal and thoracic surgery: A systematic review. Acta Anaethesiol Scand 2010;54(3):261-267.

20. Fincham JE. Response rates and responsiveness for surveys, standards and the journal. Am Pharm Educ 2008;72(2):1-3. DOI:10.5688/aj720243

21. Corner EJ, Soni N, Handy JM, Brett SJ. Construct validity of the Chelsea critical care physical assessment tool: An observational study of recovery from critical illness. Crit Care 2014;18(2):110. DOI:10.1186/cc13801 